\title{
NEUTRON DEPOLARIZATION IN SUBMICRON FERROMAGNETIC MATERIALS
}

\author{
M. Th. REKVELDT \\ Interfacultair Reactor Instituut, Technical University Delft, The Netherlands
}

The neutron depolarization technique is based on the loss of polarization of a polarized neutron beam after transmission through ferromagnetic substances. This loss, caused by Larmor precession in individual domains, determines the mean domain size, the mean square direction cosines of the domains and the mean magnetization. The method is complementary to the neutron scattering technique with respect to the size of the inhomogeneities to be determined and the dynamic range accessible. Only the static applications of the method in studying domain structures are considered. As examples will be treated metal foils under stress, oriented ferroxdur permanent magnets, soft ferrites, recording tapes and thin films. In most of these examples magnetic correlations between neighbouring domains are subject of study.

KEY WORDS Polarized neutrons, depolarization, domain size, domain orientation, stress, ferroxdur, soft ferrites, recording tapes, thin films.

\section{NEUTRON BEAM TECHNIQUES}

The applications of the neutron depolarization (ND) technique will be compared to those of the other neutron beam techniques. This is done with respect to the range of inhomogeneity sizes and the dynamic range accessible by these techniques.

Because of their wavelength and energy thermal neutron beams are especially suitable to study structure and dynamics of materials at an atomic scale by means of scattering. The technique of neutron scattering has been applied ever since research reactors came into existence to numerous materials and compounds. In addition by improving the angular resolution to about $0.5 \mathrm{mrad}$ and increasing the wavelength of the beam to above $1.0 \mathrm{~nm}$ the accessible range concerning structure by small angle neutron scattering (SANS) can be increased up to even a few tenths of a micron. With SANS the structure of a great variety of materials has been studied up to now, e.g. small particles, polymers, colloids, thin films etc. From such materials in general the size of the inhomogeneity (particle size), distribution and anisotropy of the distribution can be determined. Using polarized neutrons even the magnetic inhomogeneities can be distinguished from the non magnetic ones. For more details and applications of these techniques the reader is referred to the proceedings of recent neutron scattering conferences. ${ }^{1}$

The application of neutron depolarization (ND) ${ }^{2-19}$ starts already in 1941 by Halpern and Holstein ${ }^{2}$ theoretically and Burgy et al. in 1950 experimentally. ${ }^{3}$ 
Contrary to neutron scattering and SANS the method has never developed into a wide spread application. At present, ND is exploited at a few places in the world among which the work of Drabkin, Okorokov et al. ${ }^{4}$ and the theoretical work of Maleev ${ }^{14}$ in Leningrad and also of Badurek and Rauch et al. ${ }^{5}$ in Vienna should be mentioned. Complementary to SANS the range of sizes probed by ND is between $0.01 \mu \mathrm{m}$ and macroscopic dimensions. At the larger side of this range the application of SANS fails by a lack of sufficient resolution. However the application of the ND technique is confined to magnetic phenomena only and enables one to determine magnetic inhomogeneities as domain size, the mean square direction cosines of the domain magnetization directions and the 3D mean magnetization vector. ${ }^{6,7}$

Also in thin magnetic films ND can be applied in studying the domain structure perpendicular as well as parallel to the film. With this respect the ND is also complementary to another neutron technique which makes use of the optical properties of the neutron in reflection measurements on smooth surfaces to study the atomic and magnetic structure of the film perpendicular to the surface. In particular using polarized neutrons in this reflection technique the magnetic depth structure together with the non magnetic depth structure is obtained. ${ }^{\mathbf{8 9}}$ The application range of the reflection technique is typically from atomic dimensions up to $0.1 \mu \mathrm{m}$ perpendicular to the film, while that of the ND starts above this range however in all directions.

The dynamics in solids, liquids and gases can be studied by analysing the energy and momentum transfer by neutron scattering. The energy of thermal neutrons of about $80 \mathrm{meV}$ is of the order of the energy of the lattice vibrations and spin waves at room temperature and makes the study of dynamics at an atomic scale possible. The time scale of these processes of $10^{-12}$ to $10^{-10}$ seconds can be increased to $10^{-8} \mathrm{sec}$ using the modern neutron spin echo technique which uses Larmor precession of the neutron spin as a means of labelling the neutron with the energy it carries. The longer time scale is about proportional with the square of the wavelength of the dynamic fluctuation studied.

Also in the dynamics of materials the ND is complimentary to the techniques mentioned above. However the dynamics studied with ND happens in real time experiments in contrast with the scattering techniques. Dynamic experiments are carried out by applying a periodic action to the sample such as a magnetic field or a tension and studying the response of the domain structure on this action by measuring the neutron depolarization in periodically triggered time channels. ${ }^{10-12}$ With this technique a time resolution of about $5 \times 10^{-6} \mathrm{sec}$ can be obtained. Because of the limitation in time scale ND has only been applied on eddy current limited domain wall movements. In this paper only static applications will be presented and discussed.

In the next sections the ND technique will be treated in some detail together with a few applications in materials research under which,

-domain structure in metal foils under stress

-angular distribution of the domain magnetizations in ferroxdur

-magnetic correlations between domains in soft ferrites

- domains and magnetic correlations in recording tapes

-domain structure in thin films of $\mathrm{CoCr}$ and alumites for perpendicular recording materials. 


\section{DIMENSIONAL NEUTRON DEPOLARIZATION TECHNIQUE}

The principal set-up for ND is sketched in Figure 1. It consists of a polarizer (P) and an analyser (A) with two polarization rotators $D_{1}$ and $D_{2}$ and a magnetically shielded sample box in the centre. The devices $P$ and $A$ may be polarising crystals (e.g. $\mathrm{Fe}_{3} \mathrm{Si}, \mathrm{Cu}_{2} \mathrm{MnAl}$ ) or polarising mirrors. In the latter case a monochromator (M) is needed. The device $D_{1}$ serves to adjust the polarization of the neutron beam along one of the three orthogonal, $x, y$ or $z$ axes. Inside the polarization rotator a homogeneous magnetic field is generated in the $y-z$ plane by two coils which rotate $\vec{P}$ into the desired direction. In a second polarization rotator (similar to the first one) each component $x, y$ or $z$ of the polarization vector $\vec{P}$ can be presented parallel to the axis of magnetization of the analyser. Two guide fields $(\vec{H} \| z)$, one between the polarizing crystal and the first polarization rotator, the other between the second polarization rotator and the analyzing crystal, serve to maintain the component of $\vec{P}$ parallel to $\vec{H}$. After reflection at the analyser the neutrons are detected by a ${ }^{3} \mathrm{He}$-gas counter. The set of measured intensity $I_{i j}$ determines the so called depolarization matrix

$$
D_{i j}=\frac{I_{s}-I_{i j}}{I_{s}-I_{m}}
$$

where $i, j$ refer to the adjustment directions $x, y$ or $z$ of the two polarization rotators and $I_{s}$ and $I_{m}$ are the intensities of the fully depolarized and maximum polarized beam respectively.

The neutron flux at the sample position is about $8 \times 10^{4} \mathrm{n} \mathrm{cm}^{-2} \mathrm{~s}^{-1}$ and the flux of the depolarized beam at the detector position is typically about $2 \times$ $10^{3} \mathrm{n} \mathrm{cm}^{-2} \mathrm{~s}^{-1}$. The intensity $I_{m}$ is typically $10-20 \%$ of $I_{s}$, dependent on the

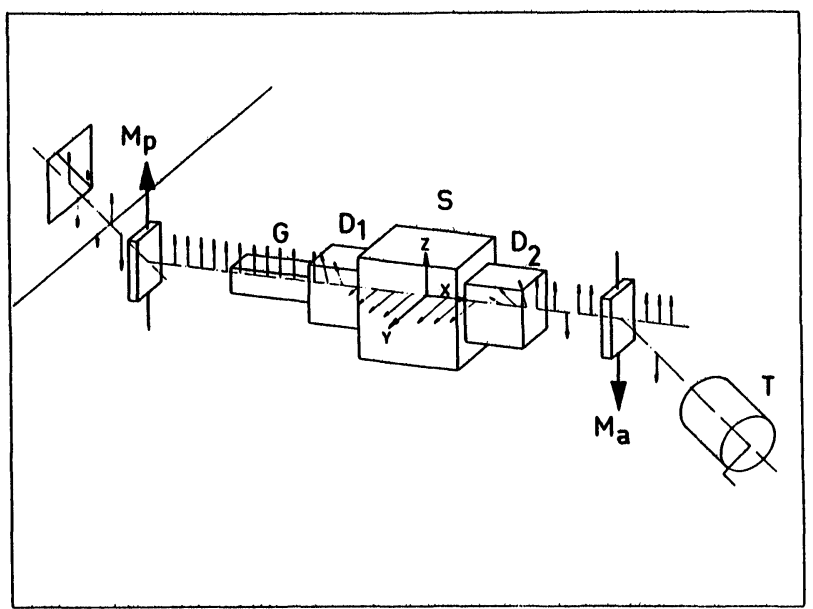

Figure 1 Sketch of a neutron depolarization set-up. The arrows indicate the state of polarization of the neutron beam. The symbols $D_{1}$ and $D_{2}$ denote the polarization rotators, $G$ the guide field and $M_{p}$ and $M_{a}$ the polarizing crystals respectively. The large arrows indicate the magnetization directions of $M_{p}$ and $M_{a}$. The sample holder and detector are denoted $S$ and $T$. 
wavelength of the neutron beam. The polarization vector of the neutron beam can be adjusted by means of an on line calibration procedure with a precision better than one degree deviation from the laboratory system of reference.

Time dependent phenomena can be studied by repeating the process (e.g. a magnetization reversal) periodically in time. In one period the intensity is counted in successive time channels of predefined length $\Delta t$. The number of measuring cycles is determined by the desired counting statistics. The time resolution is about $5 \mu$ s and results from the wavelength spread $d \lambda / \lambda$ of the beam and the time resolution of the detector.

\section{DEPOLARIZATION THEORY ${ }^{6,10}$}

\section{General}

The time behaviour of the neutron spin in a homogeneous magnetic field $\vec{B}$ is described by the classical equation of motion

$$
\frac{d \vec{P}}{d t}=\gamma(\vec{P} \times \vec{B})
$$

where the polarization $\vec{P}$ corresponds to the average spin direction of the neutrons, and $\gamma$ to the gyromagnetic ratio of the neutrons. The solution of this equation, which is fully equivalent with its quantum mechanical counterpart, is a pure rotation of $\vec{P}$ around $\vec{B}$. This rotation can be described by

$$
\vec{P}(\vec{n}, t)=D(\vec{n}, t) \vec{P}_{0}
$$

where $D(\vec{n}, t)$ is a rotation matrix describing the rotation over an angle $\omega t=\gamma B t$ around the direction $\vec{n}=\vec{B} / B$.

The polarization change by transmission through a sequence of $N$ magnetic domains can be described by a product row of rotation matrices

$$
\vec{P}_{N}=D\left(\vec{n}_{N}, t_{N}\right) \ldots D\left(\vec{n}_{1}, t_{1}\right) \vec{P}_{0} .
$$

In various magnetic systems this product row can be evaluated and leads to a theoretical depolarization matrix. This matrix is described in the domain magnetization unit vectors $\vec{n}_{N} \ldots \vec{n}_{1}$ and the interaction times of the neutrons with the successive domains $t_{N} \ldots t_{1}$, which are proportional to the domain sizes. By measuring the depolarization matrix a maximum of 9 domain parameters can be determined, corresponding to the number of matrix elements. The 9 matrix elements do not always lead to 9 independent equations in the domain parameters, so in many cases a lower number of parameters can be determined.

This theory has been applied in the study of various domain structures a few of which will be treated in the next sections.

\section{Random Domain Structures}

Evaluation of Eq. (4) in a domain structure, where the only correlation between neighbouring domains is the mean magnetization, results in a theoretical depolarization matrix described in terms of the domain quantities 
$\left(B_{s}^{2}\left(1-m^{2}\right) \delta,\left\langle B_{i}\right\rangle\right.$ and $\gamma_{i}=\left\langle B_{i}^{2}\right\rangle / B_{s}^{2}$ for $i=x, y$ and $z$. Here $B_{s}$ is the spontaneous induction and $B_{i}$ is the $i$ th component of the magnetic induction in the domain, $m$ the reduced mean magnetization $\left(m=\langle B\rangle / B_{s}\right)$ and $\delta$ the mean domain size. The $\gamma_{i}$ are the squares of the direction cosines of the magnetic induction in the domains. These quantities can be deduced from a measured depolarization matrix $D$ by means of

$$
\begin{gathered}
\left\langle B_{x, y, z}\right\rangle=\left(D_{z y, x z, y x}^{\prime}-D_{y z, z x, x y}^{\prime}\right) / c \lambda d \\
B_{s}^{2}\left(1-m^{2}\right) \delta=\operatorname{Trace} D^{\prime} / 2 c_{2} d \\
\gamma_{i}^{\prime} \equiv \frac{\left\langle n_{i}^{2}\right\rangle-m_{i}^{2}}{1-m^{2}}=1-\frac{2 D_{i i}^{\prime}}{\text { Trace } D^{\prime}}
\end{gathered}
$$

with $i=x, y, z, c=4.6 \times 10^{5} \mathrm{~T}^{-1} \mathrm{~m}^{-1} \mathrm{~nm}^{-1}, c_{2}=(c \lambda)^{2} / 2, \lambda$ the neutron wavelength in $\mathrm{nm}$ and $d$ the length of the neutron path in the sample.

Here $n_{i}$ are the reduced components of the domain magnetization and $m_{i}$ the reduced magnetization components of the sample. The matrix $D^{\prime}=-\log D$ is obtained by diagonalizing $D$ and calculating $\log D$ according to $\log D=$ $\log \left(S^{-1} D_{d} S\right)=S^{-1}\left(\log D_{d}\right) S$.

\section{Domain Structure with Correlations}

In case of correlations in the domain structure ${ }^{14}$ different from those occurring by magnetization, and which may be described by,

$$
K=\left\langle\vec{n}_{p} \cdot \vec{n}_{p+1}\right\rangle-m^{2}
$$

Eq. (5b and c) transform to similar expressions where

and

$$
\delta \rightarrow \delta\left(\frac{1+K}{1-K}\right)
$$

$$
\begin{aligned}
& \gamma_{c i}^{\prime} \rightarrow\left(\frac{1-K}{1+K}\right) \gamma_{i}^{\prime}+\frac{2 K}{1+K} \gamma_{a i}^{\prime} \\
& (i=x, y \text { or } z)
\end{aligned}
$$

Here $p$ and $p+1$ indicate two arbitrary neighbour domain positions along a neutron path and $\gamma_{a i}^{\prime}$ is the squared cosine of the direction in which the correlation occurs.

\section{Oriented Particles with Anisotropy in xy Plane}

In case of zero mean magnetization and the main axes of the domain distribution parallel to the system of axes $x, y$ and $z$, only the 3 diagonal elements of the depolarization matrix are unequal zero. This results in the diagonal depolarization matrix $D_{d}$. If the polarization directions do not coincide with the main axes of the anisotropy in domain magnetization directions, the depolarization matrix is not diagonal any more. For an angle deviation $\phi$ of the main anisotropy axis in the $x y$ plane with respect to $x$ axis, the depolarization matrix can be found by 
transformation of the system of axis over an angle $\phi$ resulting in

$$
\begin{aligned}
D & =\left|\begin{array}{ccc}
\cos \phi & \sin \phi & 0 \\
-\sin \phi & \cos \phi & 0 \\
0 & 0 & 1
\end{array}\right| D_{d}\left|\begin{array}{ccc}
\cos \phi & -\sin \phi & 0 \\
\sin \phi & \cos \phi & 0 \\
0 & 0 & 1
\end{array}\right| \\
& =\left|\begin{array}{ccc}
X-S^{2}(X-Y) & C S(Y-X) & 0 \\
C S(Y-X) & Y+S^{2}(X-Y) & 0 \\
0 & 0 & Z
\end{array}\right|
\end{aligned}
$$

For convenience the following abbreviations have been used,

$$
\begin{array}{rl}
X=D_{d x x} & S=\sin \phi \\
Y=D_{d y y} & C=\cos \phi \\
Z=D_{d z z} . &
\end{array}
$$

By measuring a depolarization matrix $D$, the deviation angle $\phi$ of the main axis of the anisotropy follows according to Eq. (9) directly from

$$
\operatorname{tg} 2 \phi=\frac{D_{x y}+D_{y x}}{D_{y y}-D_{x x}}
$$

For small deviation angles a similar expression is valid for the perpendicular direction in the $x z$-plane.

The mean square direction cosine $\gamma_{x}$ follows from Eq. (9)

and Eq. (5c)

$$
D_{d x x, y y}=\frac{D_{x x}+D_{y y}}{2} \pm \frac{D_{x x}-D_{y y}}{2 \cos 2 \phi}
$$

$$
1-\gamma_{x}=\frac{2 D_{x x}^{\prime}}{\text { Trace } D^{\prime}} \quad \text { with } \quad D^{\prime}=-\log D_{d}
$$

In case $D_{d y y}$ and $D_{d z z}$ are too small,

$$
1-\gamma_{x}=\frac{D_{x x}^{\prime}}{c_{2} B_{s}^{2} \delta d}
$$

Anisotropy in $\gamma_{i}$

Concerning the quantity $\gamma_{x}$ it should be noted that according to Refs. 6 and 15 even in a random distribution of domain magnetization orientations still anisotropy in the depolarization is found due to the demagnetizing fields of the domains which affect the polarization $\|$ and $\perp$ to the transmission direction differently. With domain particles with an anisotropic distribution of domain magnetizations, with the $x$-axis as main axis of the distribution, and no anisotropy in the $y z$-plane, this anisotropy amounts to, ${ }^{15}$

$$
\frac{\ln D_{x x}}{\ln D_{y y}}=\frac{2\left(1-\gamma_{x}\right)}{1+3 \gamma_{x}}
$$


which changes Eq. (11) into

and Eq. (5b) into

$$
\frac{1-\gamma_{x}}{1+\gamma_{x}}=\frac{2 D_{x x}^{\prime}}{\text { Trace } D^{\prime}}
$$

$$
B_{s}^{2}\left(1-m^{2}\right) \delta=\frac{\text { Trace } D^{\prime}}{2 c_{2} d\left(1+\gamma_{x}\right)} \text {. }
$$

For $\gamma_{x} \approx 1$, the quantity $\left(1-\gamma_{x}\right)$ increases by a factor 2 due to this effect, while it remains unaltered in the region of $\gamma_{x} \ll 1$. It should be mentioned that Eqs. (12 and 13) are only true when the domain distribution anisotropy is in the same direction as the transmission direction of the neutrons.

A similar method of measuring magnetic texture with polarized neutrons has also been described by Akselrod et al. ${ }^{16}$ In his method the sample has to be rotated in various directions to determine the texture, which seems to be more laborious. However, the method is based on the same physics.

\section{APPLICATIONS}

\section{Domain Structure in Metal Foils Under Stress}

To demonstrate the sensitivity of the ND technique to the domain size and local anisotropy of the domain magnetization, depolarization experiments have been carried out in $\mathrm{Ni}$ and $\mathrm{FeNi}$ foils of dimensions $(30 \times 10) \mathrm{mm}^{2}$ in the plane and a thickness of 0.125 and $0.05 \mathrm{~mm}$ respectively. A tensile stress was applied along the $y$-direction (long side of foil). The neutron beam passes along the $x$-direction, perpendicular to the foil. Before the experiments the foils were annealed at $900^{\circ} \mathrm{C}$ for $24 \mathrm{hrs}$. The experiments have been carried out without applying a magnetic field. The three diagonal depolarization matrix elements were translated in the appropriate domain parameters $\delta$ and $\gamma_{i}$ using Eq. (5b and c) and the results are given in Figure 2. The opposite behaviour of $\gamma_{y}$ in the two foils can be attributed to the opposite sign of the magnetostriction constants. In the $\mathrm{Ni}$ foil the negative magnetostriction constant favours the magnetization direction perpendicular to the pull direction while the reverse occurs in the FeNi foil. By the plastic deformation of the foil the mean domain size $\delta$ decreases strongly irreversibly and depends a little bit on whether the foil before each measurements has been demagnetized.

The higher $\gamma_{x}$ value in case of $\mathrm{Ni}$ should be ascribed to the cross contraction in the foil which causes the applied tension in the $y$-direction to be partly distributed in the $y z$-plane of the foil. A more extensive treatment of $\mathrm{Ni}$ under stress has been given in a previous paper. ${ }^{6}$

\section{Angular Distribution of Domain Magnetization in Ferroxdur}

ND has been applied in studying the magnetization distribution of the grains in a ferroxdur segment, being a part of an electromotor. The unmagnetized specimen is shaped according to Figure $3 \mathrm{~b}$ as a part of a cylinder wall with outer radius of $4 \mathrm{~cm}$, thickness $\sim 8 \mathrm{~mm}$ and a height of $4 \mathrm{~cm}$. The magnetization of the grains of $1 \mu \mathrm{m}$ size is directed radially in the average. The following questions have been considered. What is the spread of magnetization directions around the average 

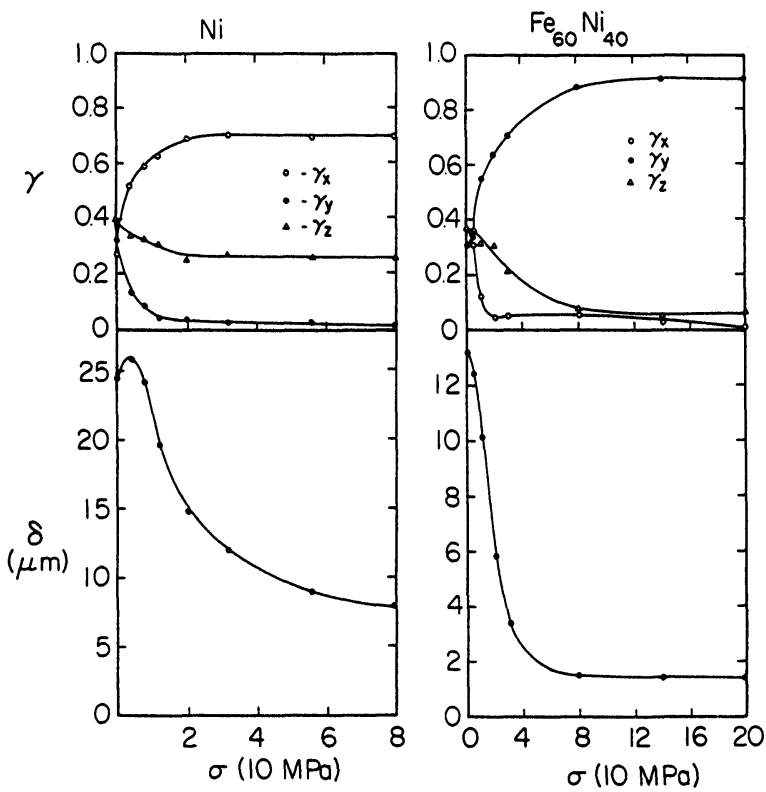

Figure 2 Depolarization results on $\mathrm{Ni}$ and $\mathrm{FeNi}$ foil as a function of the tensile stress in the $y$-direction, described in terms of the mean domain size $\delta$ and the mean square direction cosines $\gamma_{i}$ of the domain magnetizations.

radial direction and what is the deviation of this average direction from the radial direction? These problems were solved using Eqs. (14), (13) and (10) and measuring the appropriate depolarization matrix elements in a number of positions in the unmagnetized sample.

The ferroxdur sample was mounted according to Figure 1a and $b$ in an angular step apparatus in such a way that the (radial) centre of the cylindrical part of the sample coincided with the rotation axis of the angular step apparatus. In this way

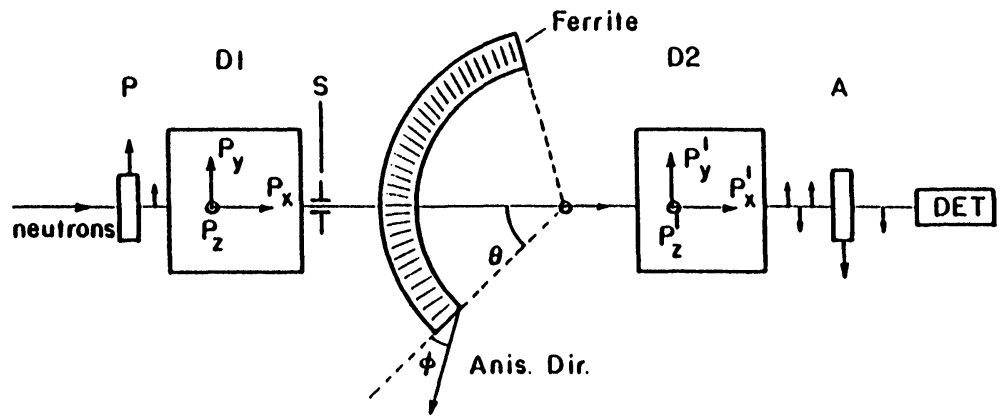

Figure 3a Top view of the set-up showing the mounting of the ferroxdur sample on an angular stepping device enabling one to scan the sample in one direction. The neutron diaphragm $S$ confines the beam to $(2 \times 5) \mathrm{mm}^{2}$. The other parts of the figure are already explained in Figure 1 . 


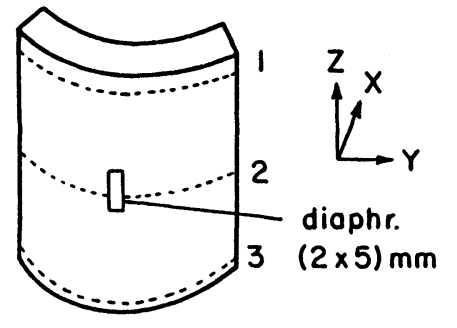

Figure 3b Sketch of the sample geometry and the positions of the sample which are investigated in the angular dependent scans.

it is guaranteed that the transmission direction of the neutron and also the $x$-axis of the laboratory system coincides with the radial direction of the sample within a few degrees. At any angular step a depolarization matrix of 5 relevant elements has been determined describing the magnetic structure in the $x y$-plane. Angular steps of 3 degrees have been made corresponding to a translation along the circumference of the cylinder of $2 \mathrm{~mm}$, which is also the size of the neutron diaphragm positioned in front of the specimen (see Figure 3a).

Three $\theta$-scans have been performed, one about $2 \mathrm{~mm}$ below the edge of the sample, one in the middle and one about $2 \mathrm{~mm}$ from the bottom. Figure 4 shows the results of one $\theta$-scan for the angular distribution width $1-\gamma_{x}$ and the angular deviation $\phi$ of the mean anisotropy axis from the radial direction. During the whole $\theta$-scan the domain size appears to be constant and equal to the mean grain size of $1 \mu \mathrm{m}$.

From the results in Figure 4a it appears that $\left(1-\gamma_{x}\right)$ is roughly constant in the material between $0.08<\left(1-\gamma_{x}\right)<0.11$ which corresponds to a cone of magnetization directions with a total top angle of about $40^{\circ}-54^{\circ}$. Only within $4 \mathrm{~mm}$ of the edge $\left(1-\gamma_{x}\right)$ increases sharply to up to 0.20 which corresponds to a cone of about $70^{\circ}$.
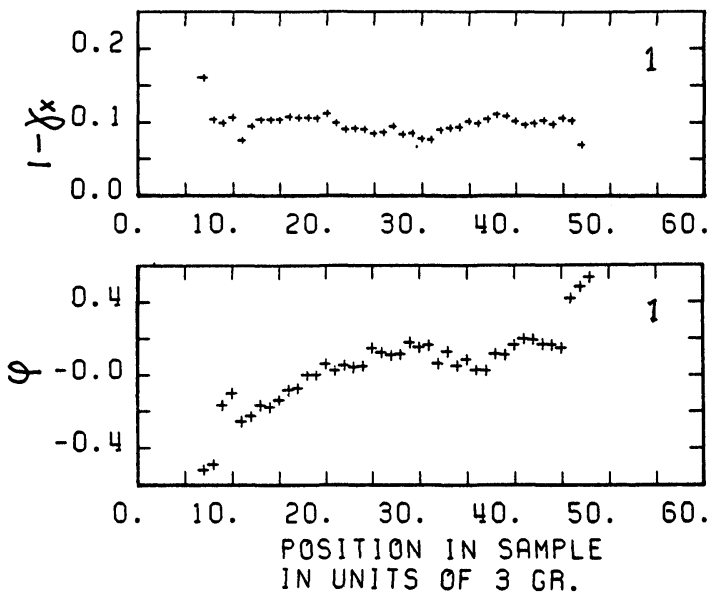

Figure 4 The angular spread $1-\gamma_{x}$ and the angular deviation $\phi$ of the mean anisotropy axis from the radial direction as a function of position. The position has been given in units of $3^{\circ}$ corresponding to steps of $2 \mathrm{~mm}$ along the surface of the sample. 
The deviation angle $\phi$ has a more irregular behaviour as a function of the position as may be seen from Figure $4 \mathrm{~b}$. Within $4 \mathrm{~mm}$ of the edge the angle $\phi$ deviates about $20^{\circ}$ from the radial direction towards the normal on the centre of the specimen. Outside these regions a gradial but also irregular behaviour as a function of position is observed. The change of $\phi$ however is rather small with respect to the total cone angle of the distribution as measured by $\left(1-\gamma_{x}\right)$.

\section{Correlations in Soft Ferrites}

ND experiments have been carried out on some hotpressed single domain partial ferrites of dimensions $(4 \times 1 \times 0.2) \mathrm{cm}^{3}$. The cross-section of the neutron beam was $(8 \times 8) \mathrm{mm}^{2}$. The main properties of the ferrites such as composition, spontaneous magnetic induction and grain size are mentioned in the Figure 5 (ferrite 1) and Figure 6 (ferrite 2). Ferrite 1 is a commercial ferrite,${ }^{17}$ with a

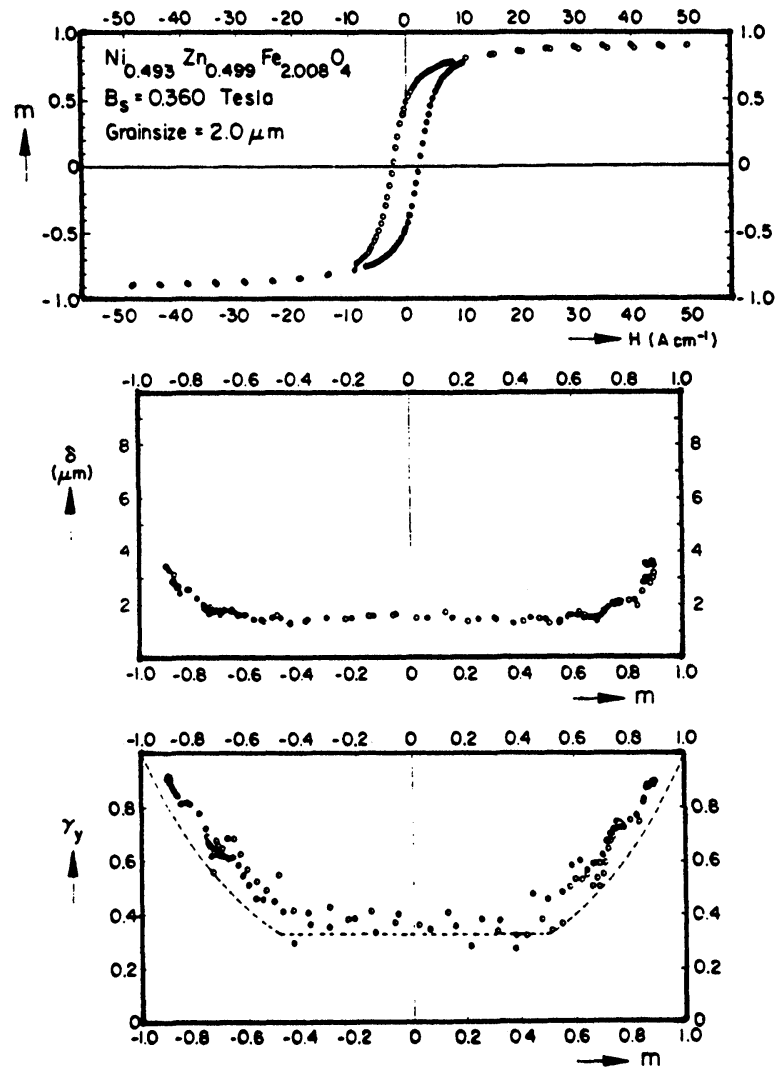

Figure 5 Neutron depolarization results on a NiZn ferrite translated into the reduced mean magnetization $m$ as a function of $H$, the domain size $\delta$ and the square direction cosine $\gamma_{y}$ as a function of $m$. The open enclosed circles in the upper and lower figures correspond to the same direction in the hysteresis curve. The dotted line in the lower figure gives the calculated result of a theoretical domain distribution. 

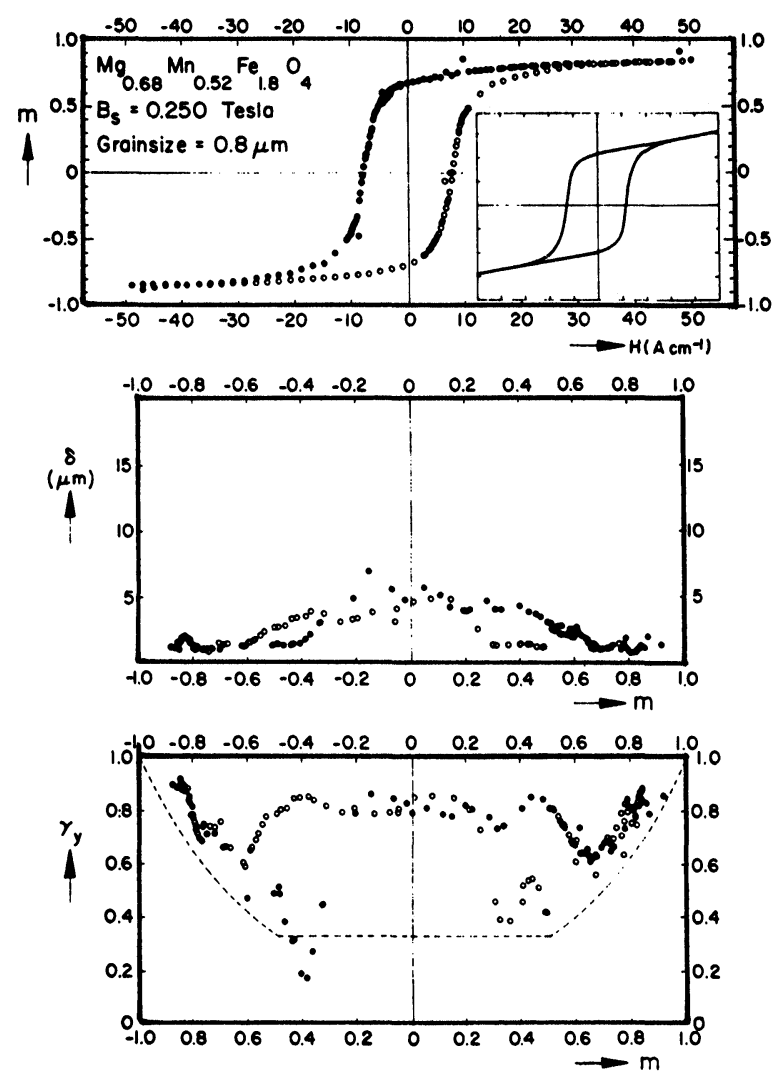

Figure 6 As in Figure 5 for MgMn ferrite.

coercive field of $2 \mathrm{~A} / \mathrm{cm}$ measured with a field amplitude of $7 \mathrm{~A} / \mathrm{cm}$. Ferrite 2 has a coercive field of $\pm 10 \mathrm{~A} / \mathrm{cm}$ measured with an induction meter at the same field amplitude as in the depolarization experiments. An insert in Figure 6 gives a complete (B-H) curve in arbitrary units obtained with an induction meter. Measurements have been performed with varying magnetic field up to $50 \mathrm{~A} / \mathrm{cm}$ in the $y$-direction. The long ends of the ferrites were fastened in a magnetic yoke to short circuit the magnetic flux of the specimen and the coil. The results have been plotted in the Figures 5 and 6, where together with the magnetization curve $\gamma_{y}$ and $\delta$ are plotted as a function of the reduced magnetization $m$. The $\mathrm{M}-\mathrm{H}$ curve in this figure has also been deduced from the depolarization data. When one accounts for the correlation between neighbouring domains, $\delta$ and $\gamma_{y}$ in the Figures 5 and 6 should be replaced by the expressions given in Eqs. (7) and (8) respectively.

The following model is used in the interpretation of the results. An isotropic distribution of domain orientations within a cone with apex angle $2 \theta$ leads to a reduced magnetization

and

$$
\begin{gathered}
|m|=0.5(1+\cos \theta) \\
\gamma_{y}=\left(4|m|^{2}-2|m|+1\right) / 3 .
\end{gathered}
$$


For $\theta=\pi / 2$ a half spherical distribution is found, corresponding to the case of remanence where $|m|=0.5$ and $\gamma_{y}=1 / 3$. If it is supposed further that the magnetization varies in the region $-0.5<m<0.5$ without changing the angle distribution of the absolute magnetization of the domains, then one obtains $\gamma_{y}=1 / 3$ in this region. A dotted line in the figures of $\eta_{y}(m)$ gives the results of this model. The results in Figure 5 for the remanence and $\gamma_{y}(m)$ can reasonably be explained by such an isotropic model. Figure 5 also shows a domain size which is nearly independent of the magnetization and of the order of the grain size of $2 \mu \mathrm{m}$. This indicates that the correlation parameter $K$ is constant for all $m$ and most likely zero on basis of its definition. Hence a correlation is very unlikely at higher values of $m$ (see Eq. (6)). The apparent increase of the domain-size for $|m|>0.7$ may be due to a systematic error in $m$. Therefore $\delta$ determined from a quantity like $B_{s}^{2}\left(1-m^{2}\right) \delta$ becomes too large if $m$ is chosen too large.

In contrast, ferrite 2 (Figure 6) shows quite a different behaviour. The remanence at zero field is about 0.7 which strongly exceeds the remanence in ferrite 1 that can be related to an isotropic distribution of domain orientations. This difference may arise when the grains in ferrite 1 have an uniaxial magnetic anisotropy whereas the grains in ferrite 2 have a polyaxial anisotropy with a preference for the easy axis nearest to the magnetization direction. Assuming further that the crystallografic direction of the grains are at random, a rough calculation of the magnetic remanences gives a qualitative agreement with the observation. The domain size $\delta$ and direction cosine $\gamma_{y}$ in Figure 3 show apparently a magnetization dependence which is ascribed to existing correlations between neighbouring domains. Near $m=0$ the correlation parameter $K=0.7$ and $\gamma_{a y}=1$ can explain the results satisfactorily. For more details the reader is referred to the original paper. ${ }^{17}$

\section{Domains and Correlations in $\mathrm{CrO}_{2}$ Recording Tapes}

The recording tape consists of a lacquer containing small magnetic particle of $\mathrm{CrO}_{2}$ needles brought on a plastic substrate. Because the magnetic domain structure and reversal mechanism are of great importance for the noise properties of the tapes, we investigated with neutron depolarization the magnetic domain distribution in this layer. The question considered was: Is the domain size in these layers the same as would be expected from the single domain particle size, which means, are the magnetic particles independent within the layer or do they show correlations by forming magnetic clusters of particles, forming apparently a much larger domain. For this purpose various depolarization experiments have been carried out, successively:

1. sample after annealing, which means thermally demagnetized;

2. sample magnetized in the length direction of the tape (z-direction) corresponding to normal use conditions;

3. sample magnetized in the plane perpendicular to $z$ (y-direction);

4. sample magnetized perpendicular to the tape plane ( $x$-direction).

It appears that the domain size in the thermally annealed state agrees very well with the diameter of the $\mathrm{CrO}_{2}$ needles on the tape of the order of $20 \mathrm{~nm}$, which should be expected theoretically. The measured angular distribution of domain 
magnetization expressed in $\gamma_{i}$ (see Eq. (5c)) corresponds to the distribution expected from magnetization measurements $\left(\gamma_{z}=0.7, \gamma_{x}=0.15\right.$ and $\left.\gamma_{y}=0.15\right)$. These values indicate a strong preference of the particle magnetizations to the $z$-direction. However in the magnetized and field demagnetized ( $z$-direction) tapes the mean domain size in the direction perpendicular to the tape is 3 to 5 times larger as the needle diameter. Because the particle size is fixed this increase is ascribed to the correlation of domain magnetization of neighbouring domains according to Eq. (7). This is an indication of the occurrence of magnetic clusters of particles in the tapes, which coherently change their magnetization direction. By varying the transmission direction of the neutrons, the size of the clusters in the $z$-direction has been determined. This size appears to be more than 20 times larger than the cluster size measured perpendicular to the tape.

By applying a field in the $y$ or $x$ direction these clusters can partly be destroyed again when the mean direction of the angular distribution within the cluster is nearly perpendicular to the magnetizing direction. Using these phenomena it seems even possible to determine the angular variations within the clusters from the measured correlation after magnetization in various directions. For more details about this procedure the reader is referred to the paper to be published. ${ }^{18}$

\section{Domain Structure in Thin Films of CoCr and Alumites used in Perpendicular Recording}

ND experiments have been performed on films of $\mathrm{CoCr}^{19}$ deposited on a Si single crystal substrate and on alumite films ${ }^{20}$ deposited on a $\mathrm{Al}$ polycrystalline substrate. Depolarization has been measured as a function of transmission angle through the film as sketched in Figure 7.

From the experiments on $\mathrm{CoCr}$ film which is known as a continuous medium it appears from $D_{x x} \approx 1$ (see Figure 8) that the local magnetization is perpendicular to the film plane. In such an uniaxial system the depolarization in the $z$-direction is simply given by

$$
D_{z z}(\theta)=\cos \phi(\theta)
$$

where $\phi(\theta)$ is the net precession angle of the polarization in the domain magnetization. At $\theta=0 \phi(\theta)$ corresponds to the rotation in the total film

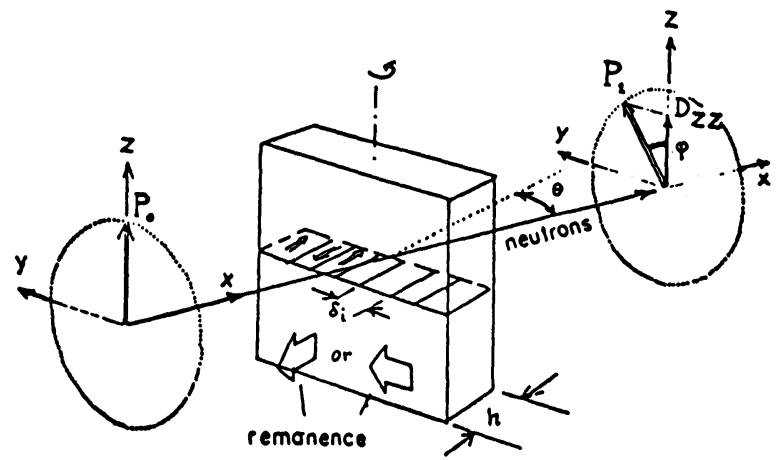

Figure 7 Sketch of the depolarization experiment on thin films of $\mathrm{CoCr}$ and alumite films. Both materials show perpendicular magnetic anisotropy as indicated in the figure. 


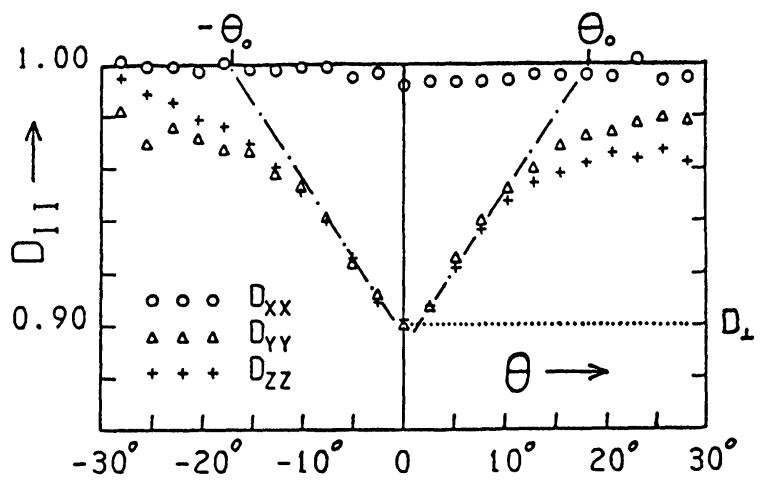

Figure 8 Behaviour of the diagonal matrix elements as a function of transmission angle $\theta$ in a stacking of $12 \mathrm{CoCr}$ films of thickness $1.9 \mu \mathrm{m}$ each.

thickness and enables one to investigate the magnetic "thickness" of the layer. This magnetic thickness can be used to test various domain models for this material, in which the closure domain structure plays an important role. This structure is responsible for the fact that the magnetic thickness is smaller than the real film thickness. With increasing angle $\theta$ the polarization experiences successive positive and negative precessions and decreases effectively with the increasing number of domain walls passed. So the angular dependence of $D_{z z}$ delivers the mean domain size in the plane of the film, which give also information about the domain model which is most applicable in this material.

Thin layers of alumite consist of iron pencils of length $t \sim 4 \mu \mathrm{m}$ directed nearly perpendicular to the film plane imbedded in an aluminium oxide layer of also $4 \mu \mathrm{m}$. In the plane the pencils are arranged in a triangular lattice. The cell size 1, pencil diameter $\delta$ and orientation distribution of the pencils were measured by electron microscopy and $X$ ray diffraction in the (111) iron reflection plane. By performing similar depolarization experiments as in the case of $\mathrm{CoCr}$ films, we have tried to get information about the domain structure in these films. Figure 9
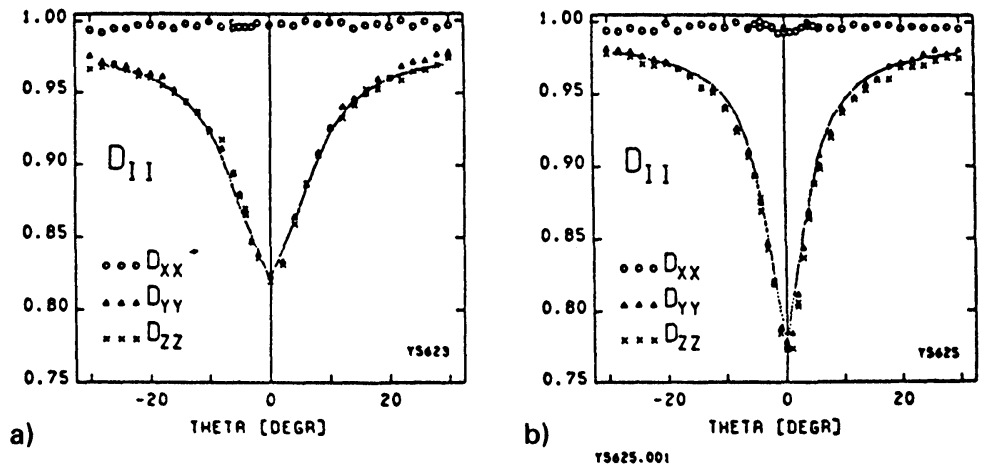

Figure 9 Behaviour of the diagonal elements as a function of transmission angle $\theta$ in two stackings of 8 films of alumites. The dotted lines represent computer simulations of $D_{z z}$ using the parameters. a) $t=4.3 \mu \mathrm{m}, \delta=42.5 \mathrm{~nm}, l=61.8 \mathrm{~nm}, k=-0.38, \Delta=0.2 \mathrm{rad}$. b) $t=4.7 \mu \mathrm{m}, \delta=42.5 \mathrm{~nm}, l=113 \mathrm{~nm}$, $k=0, \Delta=\emptyset .12 \mathrm{rad}$. 
shows the results for the depolarization matrix elements $D_{x x}, D_{y y}$ and $D_{z z}$. From $D_{x x} \approx 1$ it appears that the local magnetization indeed is perpendicular to the film plane. The data for $D_{y y}$ and $D_{z z}$, which are nearly equal in this case, have been compared with a computer simulation of the neutron depolarization using an evaluation of Eq. (16) and (7). In this particular case this yields:

$$
D_{z z}(\theta)=\left(1-N(\theta) \frac{\phi^{2}(\theta)}{2}\left(\frac{1+K}{1-K}\right)\right)^{N_{f}}
$$

Here $\phi(\theta)$ is the average precession angle in one needle as a function of $\theta$ and $N(\theta)$ is the average number of needles passed along the trajectory of the neutron. The quantity $N(\theta)$ is obtained as the product of the area $P(\theta)$ of the projection of a needle along the neutron trajectory upon the plane of the film. The quantity $1 / A_{c}$ represents the density of the needles in that plane. In formulae:

$$
\begin{aligned}
& N(\theta)=p(\theta) / A_{c} \\
& \phi(\theta)=c V / P(\theta) \cos \theta \\
& P(\theta)=\delta\left(t \tan \theta+\frac{\pi}{4} \delta\right)
\end{aligned}
$$

The quantity $K$ in Eq. (17) as defined in (6) is a correlation parameter equal to the average scalar product of neighbouring domain orientations along the neutron trajectory and $N_{f}$ is the number of films used in the experiments. Eq. (17) gives no correct description of $D_{z z}$ for small values of $\theta$. In this region Eq. (17) should be averaged over an orientation variation of the pencils described by a normalized distribution function $W(\theta)=\exp \left[-4 \theta^{2} \ln 2 / \Delta^{2}\right]$ over which an average is taken in two perpendicular directions. The result of the total simulation is shown as a dotted curve in Figure 9. Figures 9a and b correspond to alumites with pencil widths of $42.5 \mathrm{~nm}$, a thickness $4.3 \mu \mathrm{m}$ and $4.7 \mu \mathrm{m}$, and cell sizes $61.8 \mathrm{~nm}$ and $113 \mathrm{~nm}$ respectively. It appears that the most dense material (i.e. with smallest cell size) shows a rather strong correlation between neighbouring domains in the neutron trajectory which is absent in the less dense material. This difference is explained by the fact that the chance of passing neighbouring domains in the lattice in the first case is much larger than in the latter. The correlation found fits quite well with an arrangement of the domains in rows of parallel domains.

\section{SUMMARY AND CONCLUSIONS}

The ND technique and a few examples of static applications have been demonstrated. It has been shown that this technique provides a valuable extension to the applications of neutron beam techniques in materials science. The applicability lies typically in the micron region of ferromagnetic materials, just beyond the region where small angle neutron scattering finds its application. Common with the other neutron beam techniques is that bulk properties are determined. 


\section{ACKNOWLEDGEMENT}

The author should like to acknowledge W. H. Kraan, and R. Rosman for their contribution to the neutron depolarization work presented here, especially the work in the recording tapes and thin films and also for their critical comments on this paper.

\section{References}

1. Proc. Neutron Scattering Conf., Santa Fe, September 1985, Physica 136B, 1986; Proc. of Frontiers of Neutron Scattering, September 1985, Physica 137B + C, 1986.

2. Halpern, O. and Holstein, T. (1941). Phys. Rev. 59, 960.

3. Burgy, M., Hughes, D. J., Wallace, J. R., Heller, R. B. and Woolf, W. E. (1950). Phys. Rev. 80, 953.

4. Drabkin, G. H., Zabidarov, E. I., Kasman, Ya. A., Okovokov, A. I. and Trunov, V. A. (1965). Sov. Phys. JETP 20, 1548. Maleyev, S. V., Runov, V. V., Okorokov, A. I. and Gukasov, A. G. (1982). J. de Physique 43, C7-83.

5. Rauch, H. (1966). Z. Physik, 197, 373; Rauch, H. und Löffler, E. (1968). Z. Physik, 210, 265; Badurek, G., Janeschitz, G., Weinfurter, H., Hammer, J., Rauch, H. and Steiner, W. (1982). J. de Physique, 43, C7-57; Veider, A., Badurek, G., Grössinger, R. and Weinfurter, H. Proc. Conference on Magn. Recording Materials, Salford, September 1987.

6. Rekveldt, M. Th. (1973). Z. Physik, 259, 391.

7. Okorokov, A. I., Runov, V. V. and Gukasov, A. G. (1978). Nucl. Instr. and Methods, 157, 487.

8. Felcher, G. P., Gray, K. E., Kampwirth, R. T. and Brodsky, M. B. (1986). Physica, 136 B, 59. Felcher, G. P., Hilleke, R. O., Crawford, R. K., Haumann, J., Kleb, R. and Ostrowski, G. (1987). Rev. Sci. Instrumen., 58, 609.

9. Maykrzak, C. E., (1986). Physica, $136 \mathrm{~b}, 69$.

10. Rekveldt, M. Th. and van Schaik, F. J. (1979). J. Appl. Phys., 50, 2122.

11. van Schaik, F. J., Burgmeyer, J. W. and Rekveldt, M. Th. (1981). J. Appl. Phys., 52, 352.

12. van Schaik, F. J., Rekveldt, M. Th. and van Dijk, J. W. (1981). J. Appl. Phys., 52, 360.

13. Stüsser, N. and Rekveldt, M. Th. (1988). J. Appl. Phys.

14. Rekveldt, M. Th. (1976). J. Magn. Magn. Mat., 1, 342.

15. Maleev, S. V. (1982). J. de Physique, 43, C7-23.

16. Akselrod, L. A., Gordeev, G. P., Lazenbnick, J. M. and Lebedev, V. I. (1979). Nucl. Instr. and Meth., 164, 521.

17. Rekveldt, M. Th. (1977). J. de Physique, 38, C1-23.

18. Rosman, R., Rekveldt, M. Th. and Cramer, H. contribution to ICM, Paris, July 1988.

19. Kraan, W. H., Rekveldt, M. Th., Hemmes, K. and Lodder, J. C. (1987). IEEE Transactions on Magnetics, Mag., 23, 65.

20. Kraan, W. H., Rekveldt, M. Th., Umahara, Y. and Tokushima, T. (1988). IEEE Transactions on Magnetics, 24, 1793. 